\title{
Time-Dependant Illumination and Animation of Virtual Hagia-Sophia
}

Alessandro E. Foni, George Papagiannakis, Nedjma Cadi-Yazli and Nadia Magnenat-Thalmann

This paper presents a case study centered on the virtual restitution and virtual life simulation of a highly complex and endangered heritage edifice: the church of Hagia Sophia, in Istanbul, Turkey. The goal of this article is to describe the techniques used in order to achieve a real time rendering and animation of the selected space and its characters, as well as to point out the challenges and solutions that such a work implies at different stages in production. Most of these issues are focused on the reconstruction of the architecture of the site; however, in order to achieve an accurate simulation, the social aspect is not to be omitted. The importance of a heritage site resides as well in the historical characters and the social interactions that were taking place there: this information allows a better understanding of the function and the importance of the selected site in connection with the cultural aspects of the life at a certain time. In order to strengthen the feeling of immersion in a heritage edifice virtually restituted, it is important to recreate virtual life and describe the timely evolutionary aspects of the edifice as well. 


\section{Introduction}

Building a 3D model of a complex heritage site for a virtual reality simulation may become a difficult task when it comes to dealing with computer graphics hardware limitations. Therefore, specific considerations on the intended final use of the simulation should be made in order to provide a model satisfying the particular limitations of a selected support. For example, if the user experience should be limited to a passive walk-trough, more efforts can be concentrated to reproduce precisely all the complexity of the site in a non interactive simulation; inversely, if the user should be allowed to freely explore the virtual space in a 3D real-time environment, a certain balance between the fidelity of the visual reconstruction and the weight of the model ought to be found.

To achieve a successful realistic 3D simulation of a selected heritage site, different aspects are therefore critical: the choice of the appropriate modeling techniques, the preparation of the lighting models and the texture creation process (which heavily contributes to the surface appearance representation). All these aspects are even more important when the selected space should be prepared for a real-time platform, where issues on the trade-off between frame-rate, and geometrical accuracy of the model must be considered.

The selected site has been chosen for its geometrical complexity and for its importance as landmark in both the Byzantine, Islamic and world ecclesiastical architecture (the edifice was added in 1996 in the annual list of endangered monuments by the World Monuments Watch). The history of the building and the subsequent modifications (cathedral, mosque and museum) allowed us to perform a virtual simulation of the restituted space and its evolution through time. The main part of this work describes the virtual restitution of the site visualized as a mosque of the $\mathrm{XVI}$ century and as a Cathedral of the XI century.

\section{I.I. Character-based virtual cultural heritage}

Virtual Reality and its concept of cyber-real space invoke such interactive digital narratives that promote new patterns of understanding. The word "narrative" refers to a set of events happening during a certain period of time and providing aesthetic, dramaturgical and emotional elements, objects and attitudes [I]. Mixing such aesthetic ambiences with virtual augmentations and adding dramatic tension, can develop these narrative patterns into an exciting new edutainment medium. The abandonment of traditional concepts of static cultural artifacts for an interactive, augmented historical characterbased event representation is an important component of this redefinition: such a transition is attempted here with the real-time ceremonial visualization in a world acclaimed cultural heritage site, such as the Hagia Sophia edifice. In the upcoming sections, the Cultural Heritage Hermeneutical motivation is presented followed by the previous related work in the virtual heritage field.The real-time character simulation section presents the virtual 
character simulation technologies, the architectural evolution of the edifices and the real-time VR architecture framework implemented. Finally, the immersive real-time VR application is described followed by the concluding remarks.

\section{I.2. Virtual cultural heritage hermeneutics}

What consequences flow from the premises at the level of such 'Intangible cultural heritage' such as a Namaz Pray of an Ottoman Imam? Hermeneutics teaches us that all meaning is context-dependent and therefore unstable. The resulting conception of culture is thus poetic and creative rather than ethical or epistemological. Thus, such Heideggerian [2] notions invite us to transform and to appropriate creatively the contents of cultural traditions towards a conception of culture as a realm of unstable and indeterminate possibilities waiting for further exploration and new interpretative horizons. We may actually say that cultural ideal consists basically in an endless process of active and creative interpretations of past productions. The cultural tradition is conceived as a reservoir of living forces that can be experienced only if we free them from the petrifaction exerted during the centuries. Such living forces are the characters of ancient ceremonies, the associated time-context and specific function of an edifice etc.

Such 'interactive contextualized narratives' and 'hermeneutic experiences' are in fact stirring the fleeting notion of history and timetravel, based on the new emerging cultural fabric of the 2 Ist century. That results in intriguing possibilities for new digital narratives that will pervade the information age raising new issues on cultural heritage representation, space, time, interpretation, interaction, identity and the real.

\section{Previous work}

A Virtual Reality-based heritage experience shall ideally give to the visitor the opportunity to feel that they are present at significant places and times in the past, stimulating a variety of senses in order to allow the experiencing of what it would have felt like to be there. However, a review of the range of projects described as Virtual Heritage [3]-[I I] shows numerous examples of virtual reconstructions, virtual museums, virtual tours, and immersive virtual reality systems built as reconstructions of historic sites but sterile and devoid, or with limited inclusion, of any life forms or engaging characters that are needed to succeed an interactive experience. The main reason for their slow adoption is due to the lack of interesting interaction paradigms for character-based installations and the lack of effective frameworks for multidisciplinary synergies and common understanding between historians, architects, archaeologists and computer scientists. In this article we attempt to address such issues through the presentation of a complete methodology that has been applied to recreate a 3D interactive 
real-time virtual reality simulation with the inclusion of animated virtual human actors (exhibiting personality, emotion, body and speech simulations) that are re-enacting staged storytelling scenarios. Although initially targeted at Cultural Heritage Sites, the illustrated methodology is by no means exclusively limited to such subjects, but can be extended to encompass other types of applications. Hence, we stress that with the assistance of a modern real-time VR framework, suited for the simulation and visualization of interactive animated believable and compelling virtual characters, such virtual experiences can be significantly enhanced and that the presented content might greatly benefit from the inclusion of a dramatic tension and with the implementation of a scenario based interaction.

\section{Historical information}

Hagia Sophia (exterior depicted in Figure I) represents the apotheosis of Byzantine art and wealth [12]. Lying at the very heart of one of the richest in cultural artifacts cities in the known world, Istanbul, Turkey, Hagia Sophia's rich interior reflects more than fourteen centuries of continuous use first as an orthodox church, then as catholic cathedral, then as mosque, and finally as a secular museum.

Figure I: Left: Exterior photograph Right: Lithography by the Fossati brothers [13].

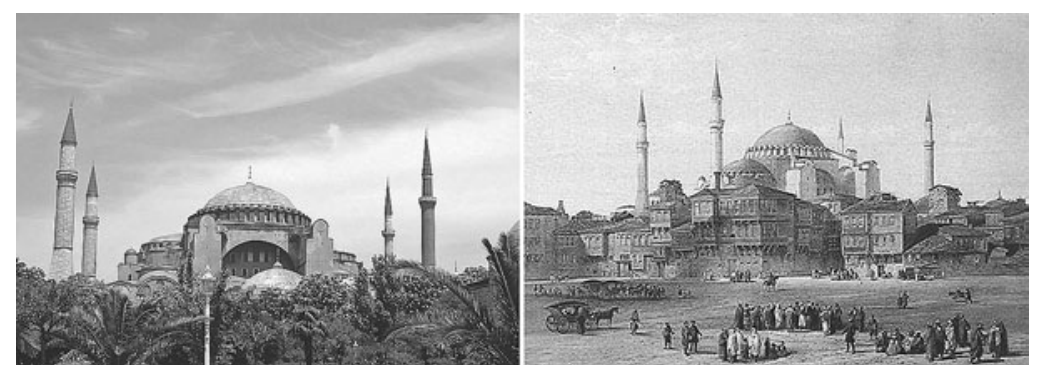

The Church of Hagia Sophia was also the Cathedral of the Ecumenical Patriarchate of Constantinople for more than one thousand years. Originally known as the Great Church, because of its large size in comparison with the other churches of the then Christian World, it was later given the name of Hagia Sophia, the Holy Wisdom of Christ, the second person of the Holy Trinity.

Justinian conceived the grandiose project of rebuilding the Great Church from its foundations. Construction work lasted five years (532-537) and in 537 Patriarch Menas consecrated the magnificent church. By an unprecedented combination of structured forms, Justinian's architects (Anthemios and Isidoros) created an immense interior of wide-spanning vaults and subtly interpenetrating spaces that had never been surpassed. 


\section{Methodology}

\section{I. Preparatory phase, data acquisition}

All the phases of the constitution of the 3D model were based entirely on two-dimensional archeological, cultural, architectural and photographic data. The data used in the preparatory phase has been collected mainly from two different sources:

I. The Yildiz Technical University of Istanbul provided all the cultural and archeological data, as well as the architectural plans and sections (shown in Figure 2) that were used as a starting base to build the $3 \mathrm{D}$ model of the $\mathrm{XVI}$ century mosque and at a later stage to virtually restitute the site as a Cathedral of the XI century. Precise measurements were performed, according to these plans, to retrieve the exact dimensions of the main structural elements and were then used in a commercial 3D software package in order to recreate these elements with the maximum precision allowed by the scale factor of the plans.

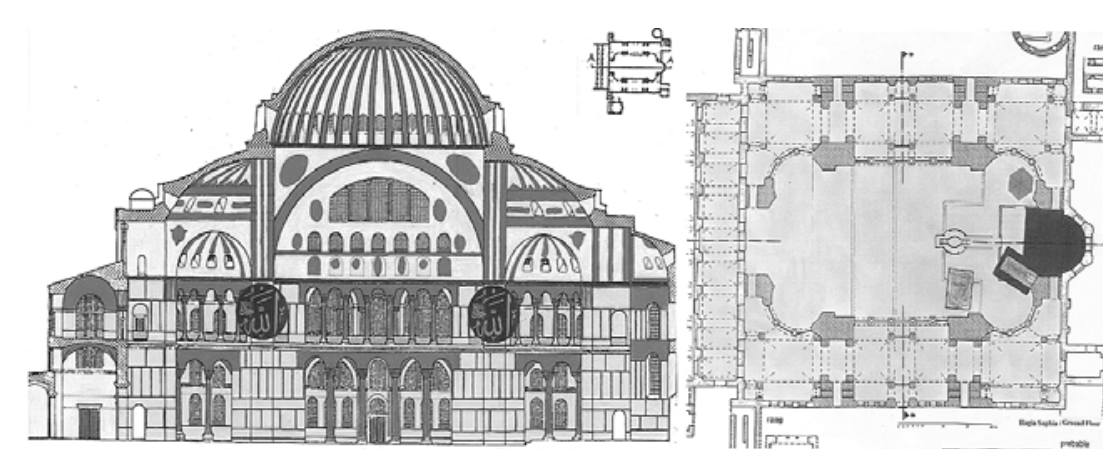

2. Since most of the available plans used a large scale factor, between $\mathrm{I}: 200$ and $\mathrm{I}: 100$, high-resolution photographic and video data (samples are shown in Figure 3) were collected on site with digital cameras. The digitally acquired data has been used as support to model elements not present on the architectural plans due to the scale factor, or to add detail to secondary and small structures that were poorly documented or omitted on the general plans of the building.

All the photographic data has been also used in a later stage as a starting base for the construction and virtual restoration of the diffuse textures.

Alessandro E. Foni, George Papagiannakis, Nedjma Cadi-Yazli and Nadia Magnenat-Thalmann 
- Figure 3: On-site interior photos.

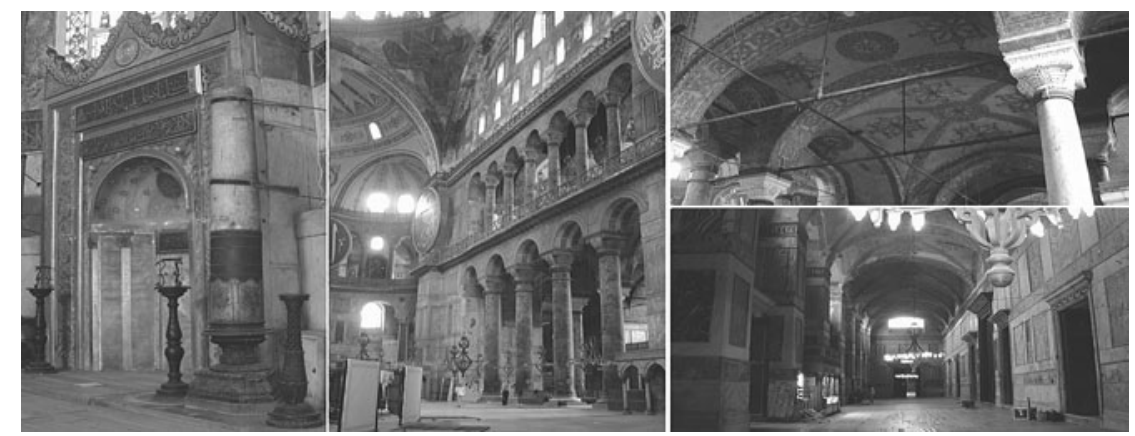

\subsection{D modeling}

Different solutions for the creation of the 3D model of the site were considered but discarded mainly because they would have implied less control over the meshes and the generated number of polygons. Laser scanning was considered but discarded for those reasons. Furthermore, its adoption would have been impractical due to the lighting conditions of the selected space and its reflective surface proprieties, and because it would have been necessary to establish far too many measurements points due to the complexity of the building and its numerous occlusions. Photogrammetric approaches have been also discarded because in those conditions they would have produced non-exploitable results. Thus, 3D Studio MAX software package was selected for the conduction of the $3 D$ virtual restitution using polygonal modeling techniques (general view depicted in Figure 4): NURBS modeling was not considered mainly because the final model is also to be used in a real-time environment.

Time-Dependent Illumination and Animation of Virtual Hagia-Sophia 289

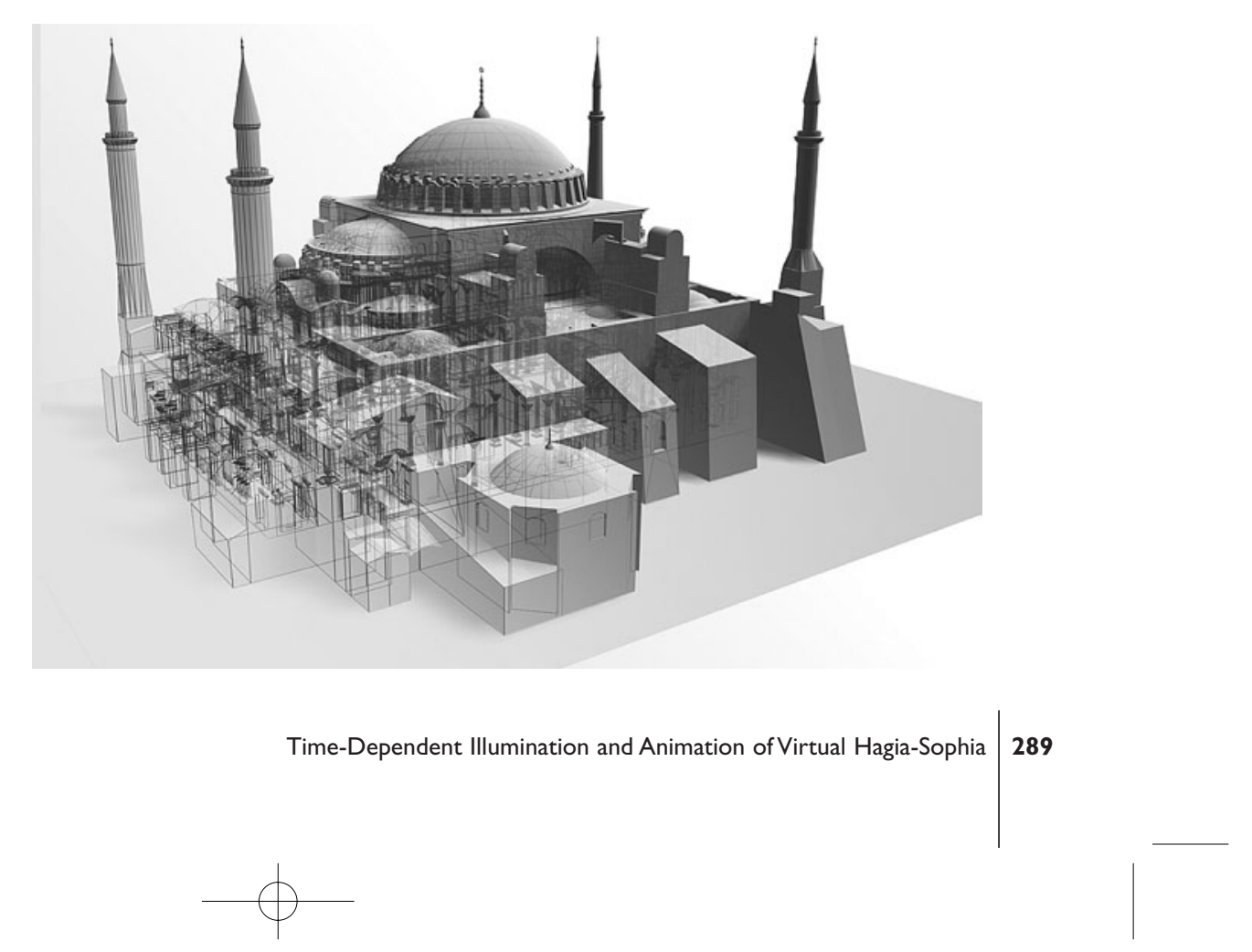

- Figure 4: View of the 3D model with wire-frame details. 


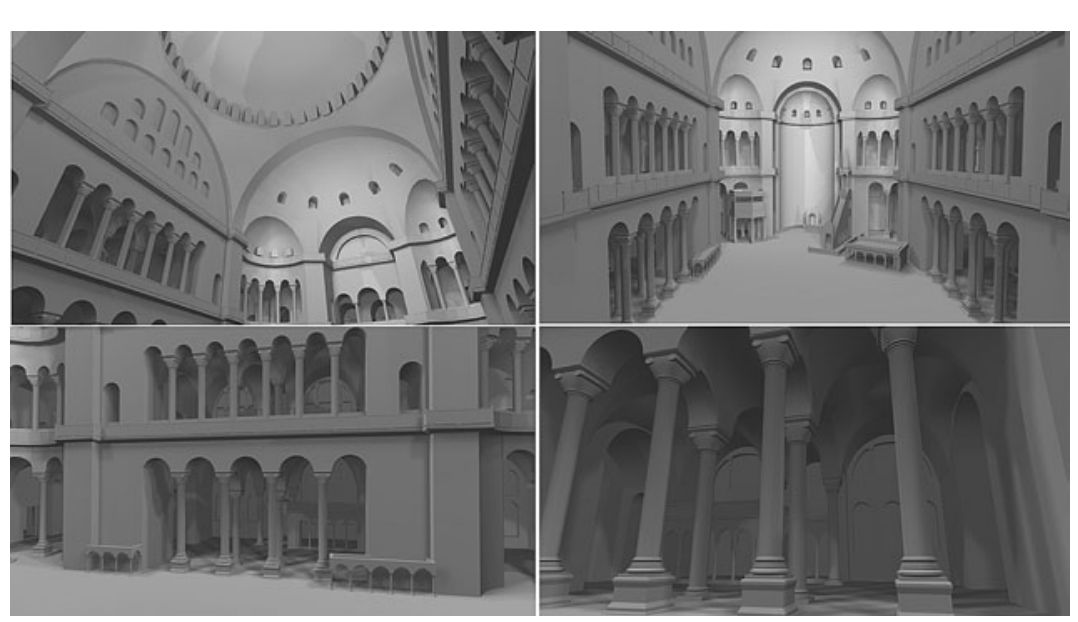

4 Figure 5: Non-textured 3D model of the interior.

Since the final 3D model (Figure 5 shows some samples of the interior) is tailored for two different applications, namely a real-time simulation and a non real-time simulation, a balance between the requirements of these two outputs was required. In order to face the challenge of such a complex heritage site, and to prepare it for a real-time simulation, different techniques were used:

3. The model was split in seamlessly overlapping modules that were separately exported to our real time platform and connected with portals in order to achieve a better frame-rate.

4. Larger textures ware down-sampled to reduce their size and their loading time.

5. Special care was given to the mesh creation of the critical elements such as the crossing vaults or the chaplets in order to keep the polygon count as low as possible without lowering the visual accuracy of the virtual restitution. To achieve such results many elements were modeled, edited and placed point by point, manually creating the desired facets and adding smooth operators where necessary. To cope with the high visual quality expected from a non real-time simulation, highly detailed versions of the textures were mapped on the surfaces of the model (details are described in section 4.3), and special attention was given to the 3D modeling of the main elements and details of the larger surfaces and structures.

\subsection{Texture reproduction}

Digital photographic acquisition of the appearance of the surfaces of the building was conducted on site. Due to lighting condition inside the building, its large dimensions and its variable state of degradation (shown in Figure 6), ranging from well preserved to highly damaged, only a small part of the collected material was finally directly exploitable for a proper virtual restitution of the selected space.

Alessandro E. Foni, George Papagiannakis, Nedjma Cadi-Yazli and Nadia Magnenat-Thalmann 
Figure 6: Details of the state of preservation of the surfaces inside the building.

Figure 7: Top: Virtually restored textures. Bottom: Acquired textures.

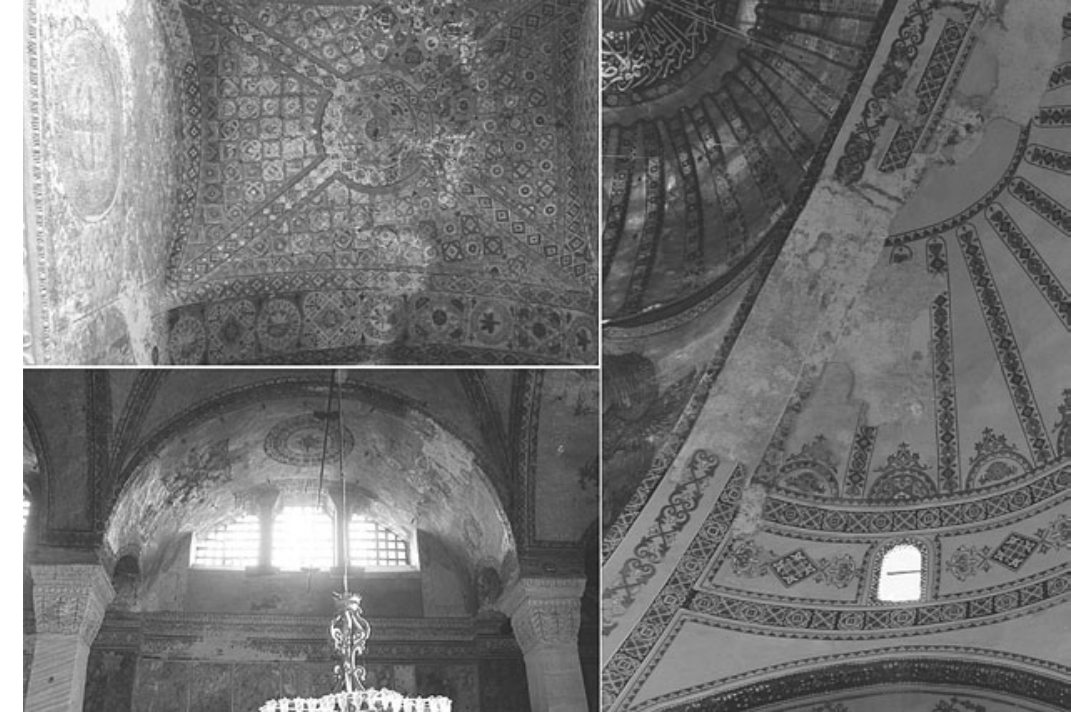

Depending on the quality of the collected material, and of the specific surface acquired, two methods were used in order to create the material textures to be mapped on the 3D model: virtual texture restoration or texture composition.

\section{Virtual texture restoration}

Virtual restoration was conducted mainly for the decorative elements severely damaged through time or for the acquired textures that were presenting heavy distortion due to the curvature of the corresponding surface during the data acquisition phase, such as the vault's decoration or the main dome's decorative elements.

Furthermore, virtual restoration was applied wherever elements belonging to a different epoch had to be removed, such as Christian decorations appearing under Islamic ones (sample results shown in Figure 7).
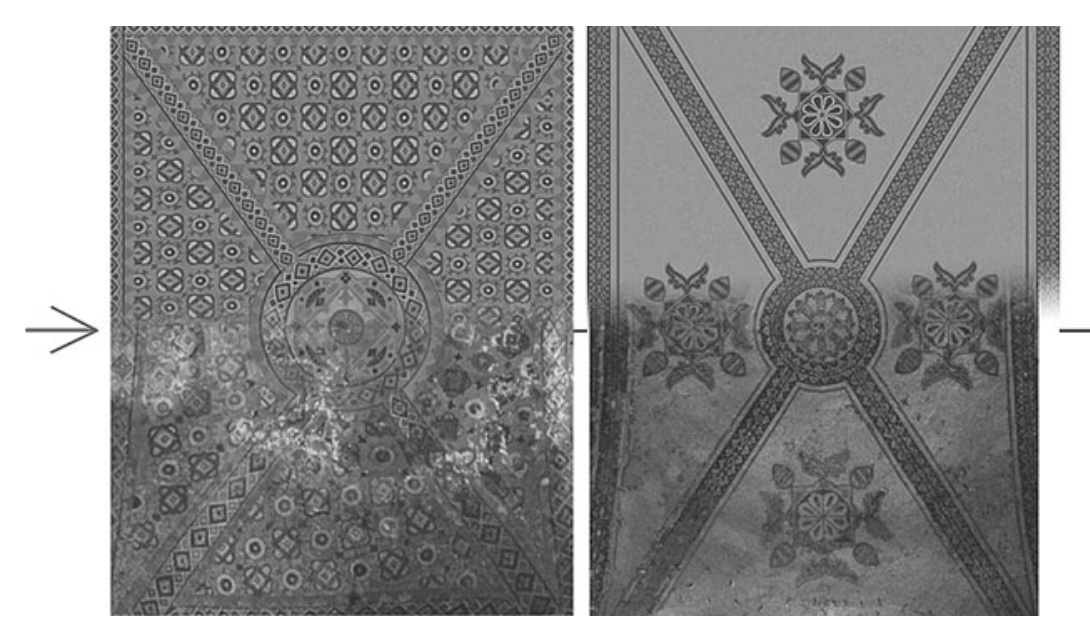

Time-Dependent Illumination and Animation of Virtual Hagia-Sophia 29 291

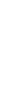


The virtual restoration process, as well as the texture composition process detailed in the next subsection, was conducted using the Adobe Photoshop 2D software package. With the support of the light and color specifications of the materials measured on site, an effort to retrieve the original color information of the missing elements was attempted. Manual editing of the acquired textures was conducted to clean the images, resaturate the colors, and fill the missing parts where excessive degradation occurred. The virtual restored versions of the acquired textures were prepared considering the needs of the UV mapping definition to be applied on the 3D surfaces (a screenshot of the textured model is shown in Figure 8).

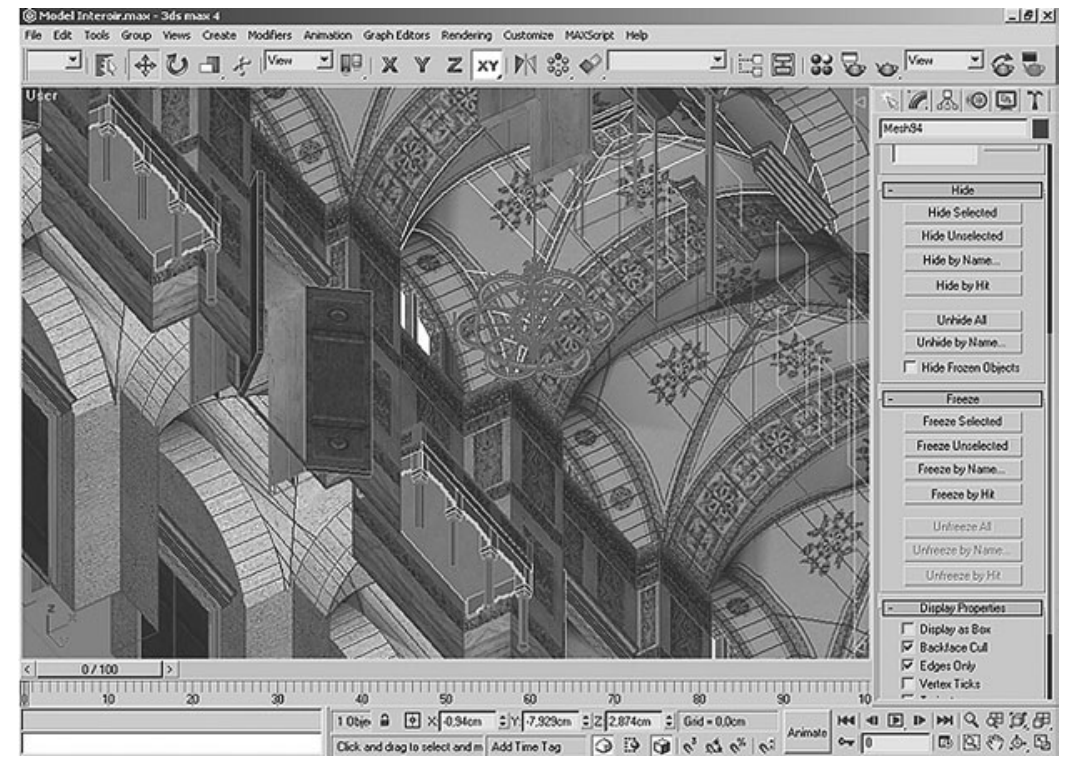

Texture composition

Texture composition was mainly conducted on the acquired elements that present an acceptable conservation state, such as the marbled walls and the stone columns. Using different views of the acquired material as source, 2D editing tools were used to clone, place and retouch exploitable elements in order to build a composite texture as close as possible to its original counterpart. Where necessary, the retouched image has been prepared to be tiled seamlessly on the target geometry (Figure 9 shows some samples of the composite textures vs. their corresponding source images)

All the textures produced with the aforementioned techniques have been prepared at very high resolutions, since it is necessary to have a high degree of precision in order to virtually restitute the textures to their original state in all their complexity (Figure 10 left). Furthermore, such

292

Alessandro E. Foni, George Papagiannakis, Nedjma Cadi-Yazli and Nadia Magnenat-Thalmann
Figure 8: Screenshot of the textured model during UV editing in 3D Studio $M A X^{T M}$ software package. 
Figure 9: Composed textures vs. source images.

- Figure 10: a: Texture extraction from a photographic source image and mapping to its corresponding 3D model. b: Rendering of the edifice with high resolution textures and photon traced light simulation.
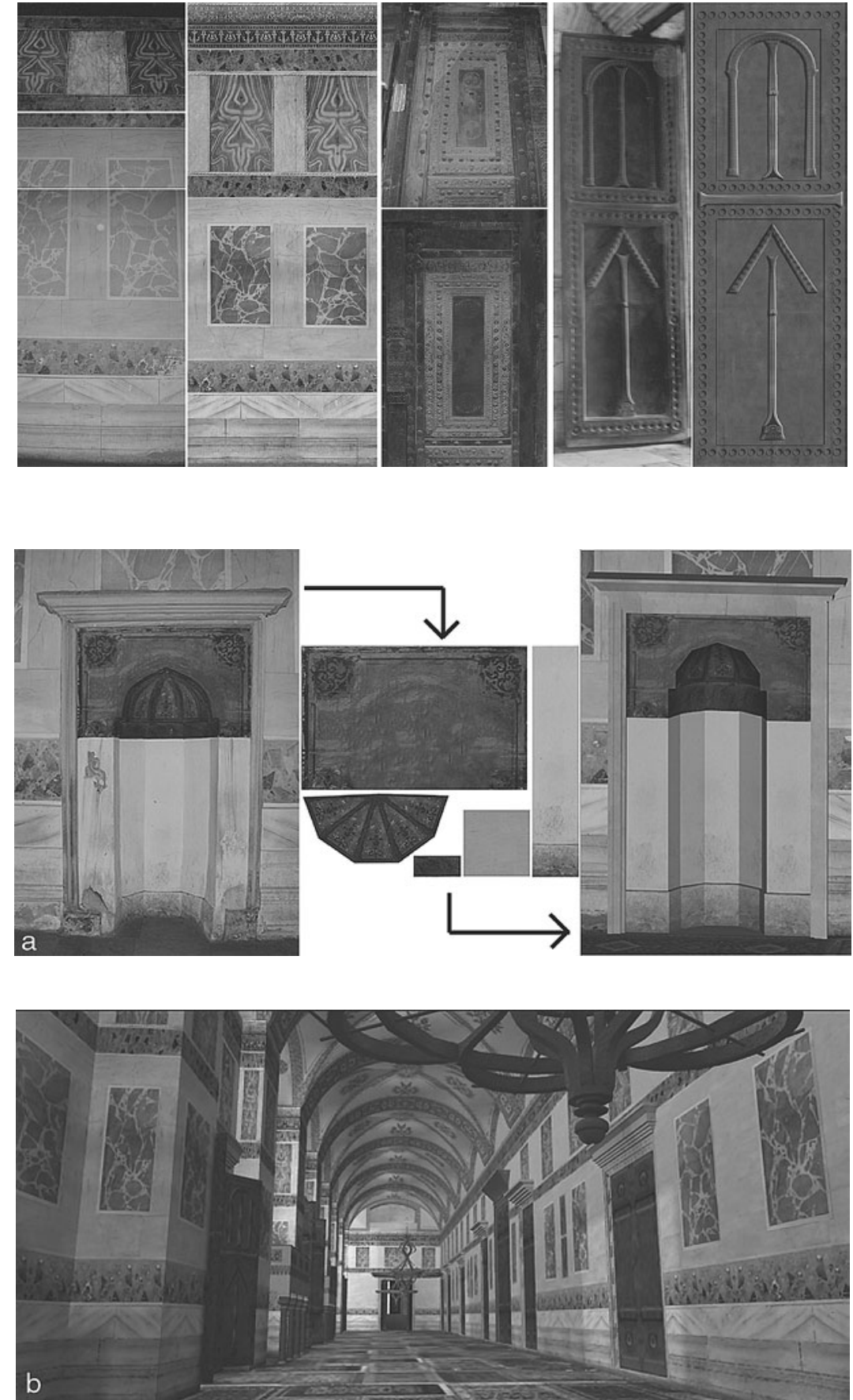

textures were used to produce high detailed renderings for the non realtime simulation of the edifice (as shown in Figure 10 right). At a later stage the same textures were down-sampled to replace the original ones in the real-time simulation.

Time-Dependent Illumination and Animation of Virtual Hagia-Sophia 293 


\subsection{Restitution of the Byzantine edifice}

The preparatory phase for the virtual restitution of the Byzantine model has been carried out by collecting all the available pertinent sources and scientific material, such as architectural and archeological plans, in addition to various works on Byzantine art and architecture. The selected sources provided precise indications for certain parts of the church and described the lost atrium along with various details pertaining to other structures lost during the edifice transformations. The 3D model of the exterior of the mosque (depicted in Figure II) was taken as a base from which all the elements that belonged to the Islamic period were removed, then such model was used as reference for the modeling of the Byzantine structures.
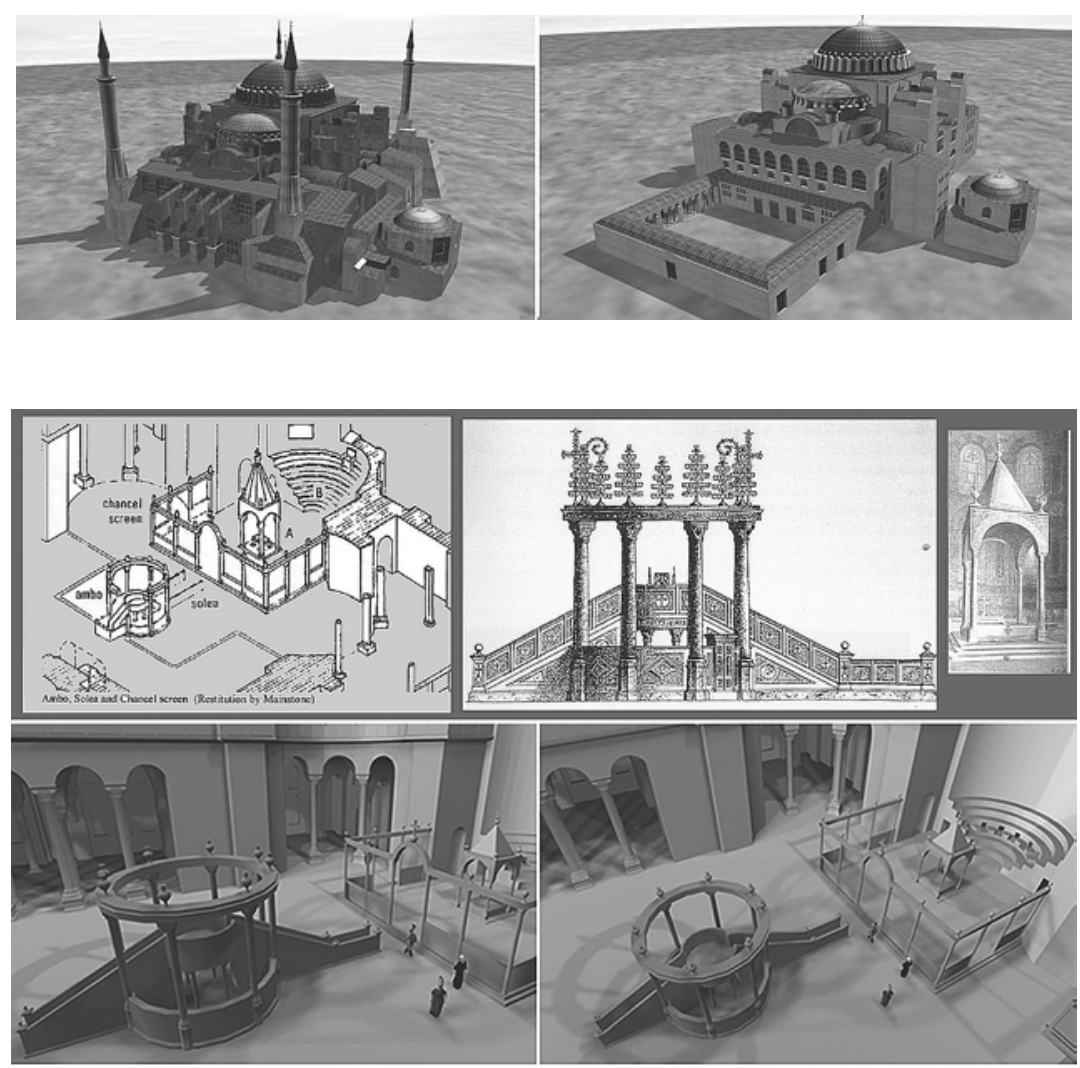

4 Figure 12: Top: Byzantine restitutions by Mainstone [14] and Antoniades. Bottom: 3D virtual restitution.
4 Figure II: Left: 3D model of the exterior of the Islamic mosque. Right: Model of the Byzantine Cathedral.

The main virtually restituted elements are the Atrium and the Byzantine liturgical furnishing (shown in Figure 12), located in the interior space of the building, such as the chancel, the ciborium, the ambo, the solea and the synthronon. The drawings and the rest of the collected scientific material, provided the necessary information relative to the arrangement and the architectural description for those parts of the edifice, and enabled us to reconstruct and position each element inside the $3 \mathrm{D}$ model.

294

Alessandro E. Foni, George Papagiannakis, Nedjma Cadi-Yazli and Nadia Magnenat-Thalmann 
Even though the aforementioned structures are long lost, enough sources were available to perform a geometrical restitution of such elements. However, no specific information was available regarding the surface proprieties and their appearance to perform an acceptable virtual reconstruction of their textures. Therefore, the reconstructed structures pertaining to the XI century Byzantine restitution were assigned a generic homogeneous surface coloring.

\subsection{Bringing the edifice to life}

In order to achieve a coherent and historically meaningful virtual visualization of any heritage site, it is crucial not only to proceed to the preparation of a scientifically correct representation of its architectural qualities, but it is equally essential to present the relations that existed between the physical site, its social function and the people that used to live at the time in which it was still in use. In such respect, it is primordial to extend the traditionally conceived virtual heritage experiences with the inclusion of historically plausible virtual humans reenacting activities typically preformed in the time dependent context of the restituted site, thus adding a human dimension to otherwise sterile simulations devoid of any social dimension. Moreover, the addition of historically consistent virtual humans into virtual reconstructions both allows for a better understanding of the use of the architectural structures present on the site, and permits the creation of more realistic simulations with the inclusion of specific ambiences, atmospheres or dramatic elements. Therefore, careful research on the clothing culture of the ottoman period was carried out and the collected data, such as patterns of different cloths (both male-female as depicted in Figure 13) and images of fabrics, were used as base for the restitution of the different types of clothes and for their simulation around the bodies of the virtual humans included in the simulation.

The virtual humans were created in order to obtain one unified and optimized model, exhibiting personality, emotion, body, cloth and speech

Figure 13: Sample images of cloths and patterns belonging to the Ottoman period.
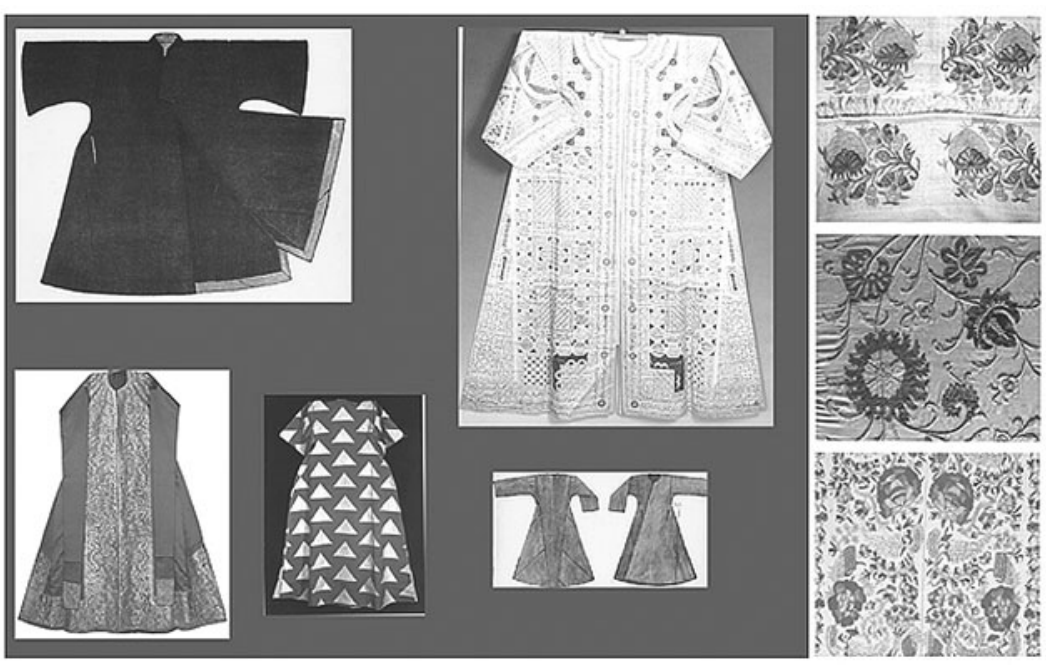

Time-Dependent Illumination and Animation of Virtual Hagia-Sophia 295 295 


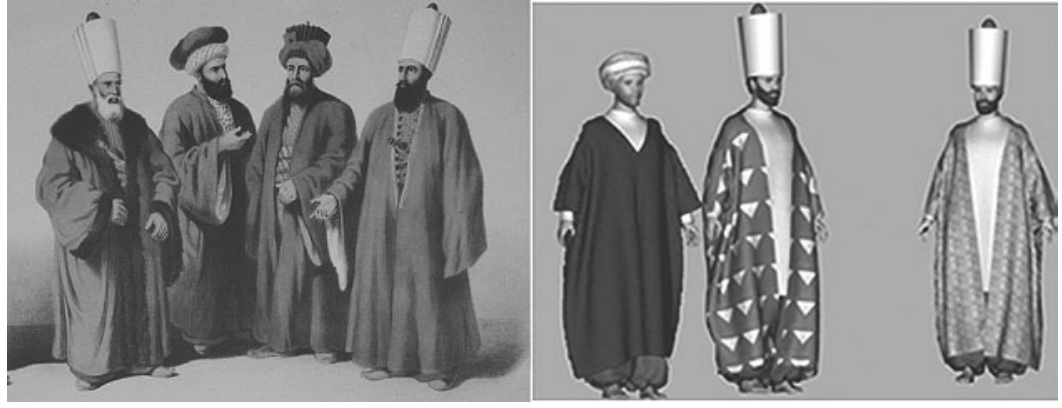

4 Figure 14: Left: Historical source images. Right: 3D modeled characters.

simulations, and featuring the ability to execute scripted staged storytelling scenarios. The implemented 3D virtual characters (examples shown in Figure 14) were also prepared while taking into consideration the total number of polygons necessary to keep a reasonable balance between the $3 \mathrm{D}$ real-time simulation restrictions and their skin deformation accuracy.

\subsection{Real-time virtual Namaz pray}

A detailed scenario for the Morning Namaz Pray as taking place in the 16th century restored Hagia Sophia edifice was prepared with the collaboration and supervision of the historians, architects and scientific personnel contributing to the project. In order to animate the virtual human reenacting such activity, a motion capture process was performed utilizing a VICON optical motion capture system. To assist the motion capturing sessions, and to provide the character animators with solid visual references and clear guidelines to prepare and time the actions performed by the virtual humans, all the events and dialogues of the scenario have been filmed and recorded while being reenacted by a real Imam. After the needed post-processing the individual movements that corresponded to the various parts of the pray were exported as separate keyframe animations ready to be applied to the $\mathrm{H}$-Anim compliant virtual characters. Finally, simulated 3D sound speeches corresponding to the verses of the pray were used to build a database of matched speeches and body animations. Thus, the 2 essential parts of the Namaz pray ceremony, were ready to be applied to the virtual Imam and the other characters to finalize the scenario based simulation.

\subsection{Evolution of the edifice throughout time}

Due to the hermeneutic need to revive the time-dependent context of the restored edifices, the need has arisen to restitute the buildings both as they were originally (i.e. as churches) and as they have been modified (i.e. as mosques). However, the Byzantine restitution of the edifices proved of high risk as not sufficient architectural-historical-archaeological data were at hand. Thus it was commonly agreed after discussions with the project cultural partners, that the Byzantine interior restitution would have depicted only

Alessandro E. Foni, George Papagiannakis, Nedjma Cadi-Yazli and Nadia Magnenat-Thalmann 
architectural modifications without image-fresco restorations (as introduced previously in section 4.4), which were unobtainable in most cases. Thus the simulations resulted as depicted in the Results section.

4.8. Rapid application prototyping using a real-time framework for VR character simulation

In order to proceed with the rapid development of such demanding high performance interactive immersive VR application, featuring advanced virtual human simulation technologies, we adopted the VHD++ real-time framework as described by [15] (overview illustrated in the following figure).

Figure 15: Concept of the generic VHD++ runtime engine with components (plug-ins).

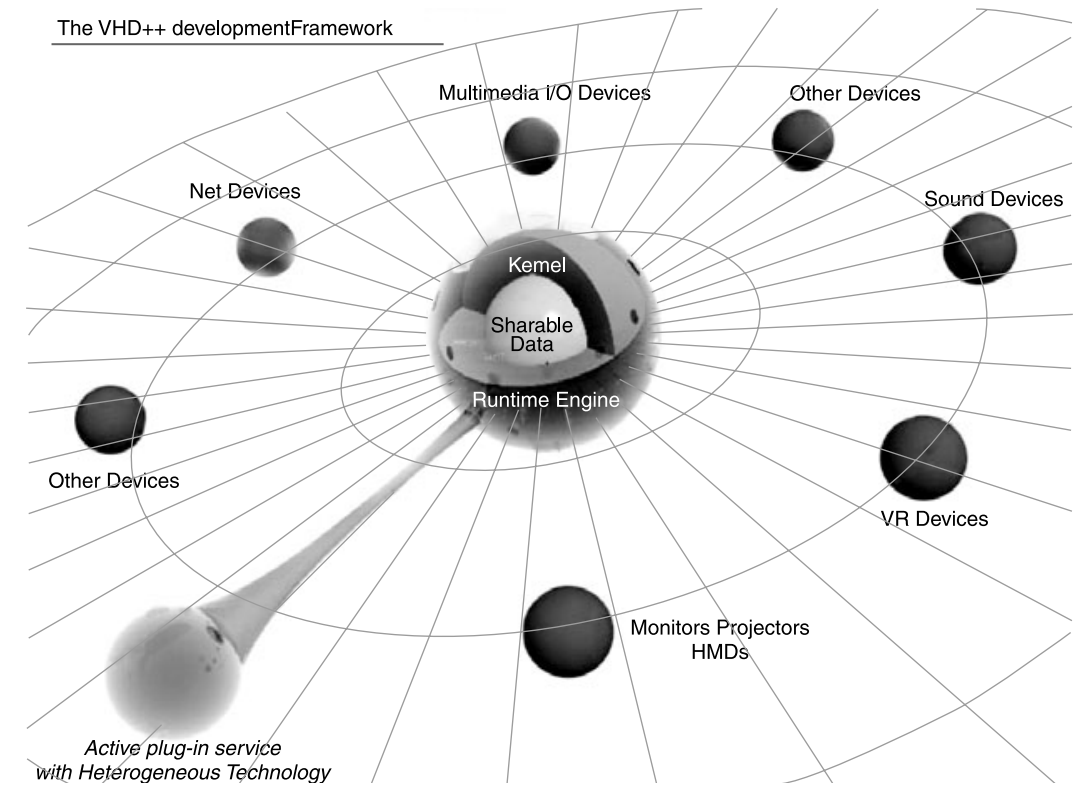

VHD++ addresses the most common problems related to the development, extension and integration of heterogeneous simulation technologies under a single system roof while combines both framework (complexity curbing) and component (complexity hiding) based software engineering methodologies. As a result, a large scale architecture and code reuse is being achieved which radically increases development efficiency and robustness of the finalVR and virtual character application. Figure I5 illustrates a graphical overview of the VHD++ framework. Without the adoption of such a framework, it would have been extremely time consuming and technically not guaranteed to successfully implement in the finalVR application all the featured heterogeneous real-time technologies, such as immersive 3D real-time graphics, 3D sound,VR interaction, virtual human animation with skinning, real-time cloth simulation, facial animation, emotion and speech. 
4.9. Real-time rendering and animation results

In this section we present some of the results achieved with the adoption of the VHD++ framework for the rapid creation of an immersive VR application prototype. A Christie Mirage 1000 wall projector was used to provide both active and passive stereo projection, as VHD++ supports sequential stereo rendering (viewed with either shutter or polarized glasses). Thus the immersive aspect of the VR simulation was ensured.The final VR application was executed in a PC system, rendering real-time environments ranging from $60 \mathrm{k}$ to $400 \mathrm{k}$ polygons, I0k-polygons humans with deformable skins, while running on a Win 2000, 2GB RAM, Pentium I.5GHz, with NVIDIA Quadro 4 980XGL graphics card, yielding $25 \mathrm{fps}$ performance.

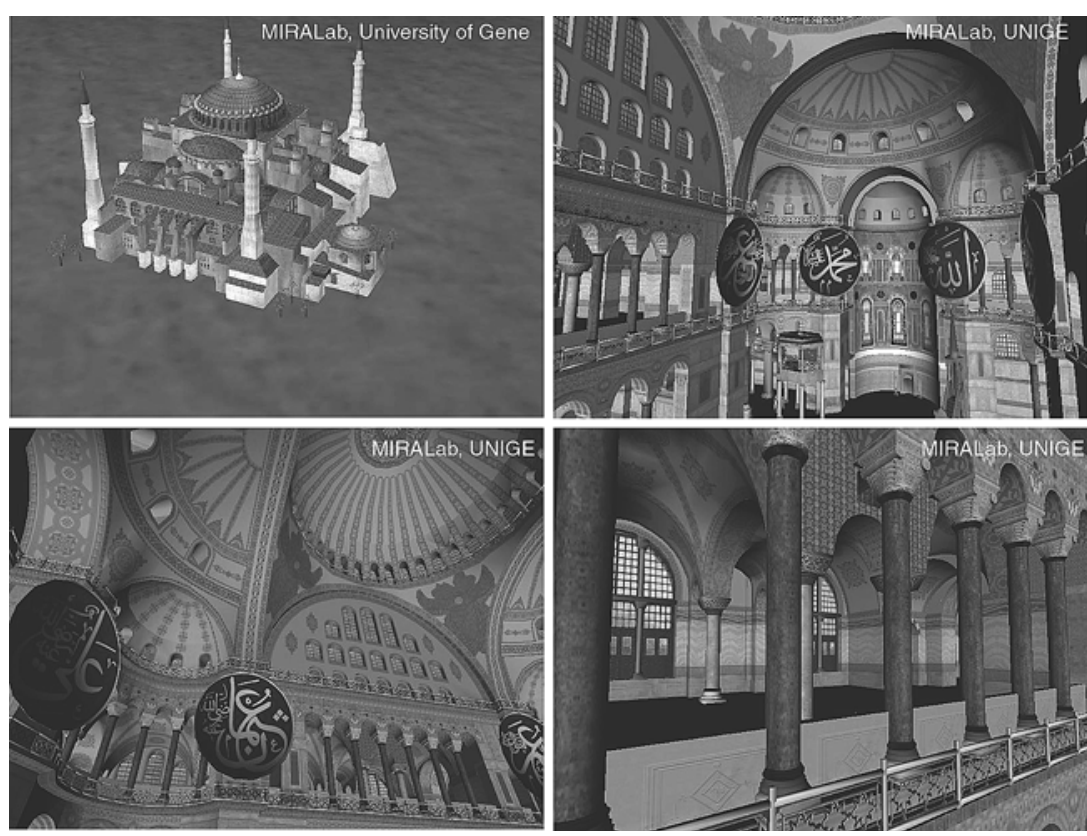

4 Figure 16: Real-time visualisation of the Hagia Sophia Ottoman Mosque.
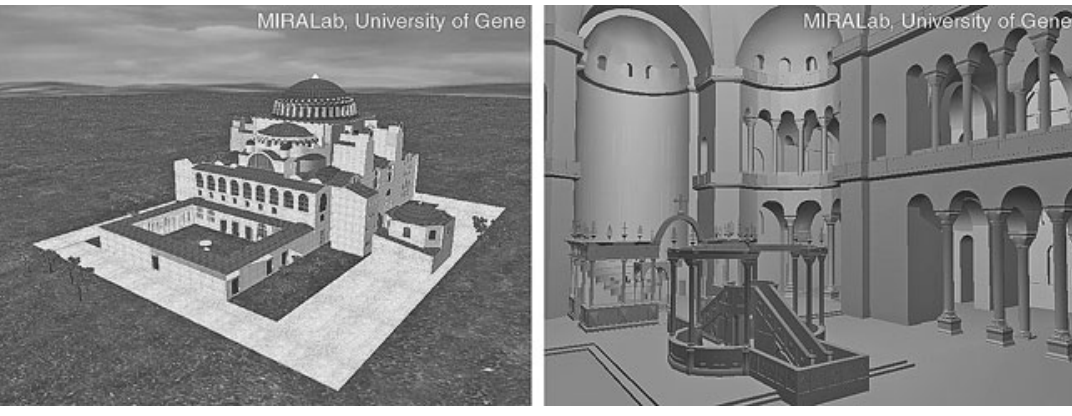

4 Figure 17: Real-time visualisation of the Hagia Sophia Byzantine Church.

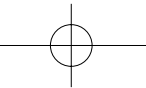


- Figure 18: Real-time virtual Imam reenacting the Namaz Pray.
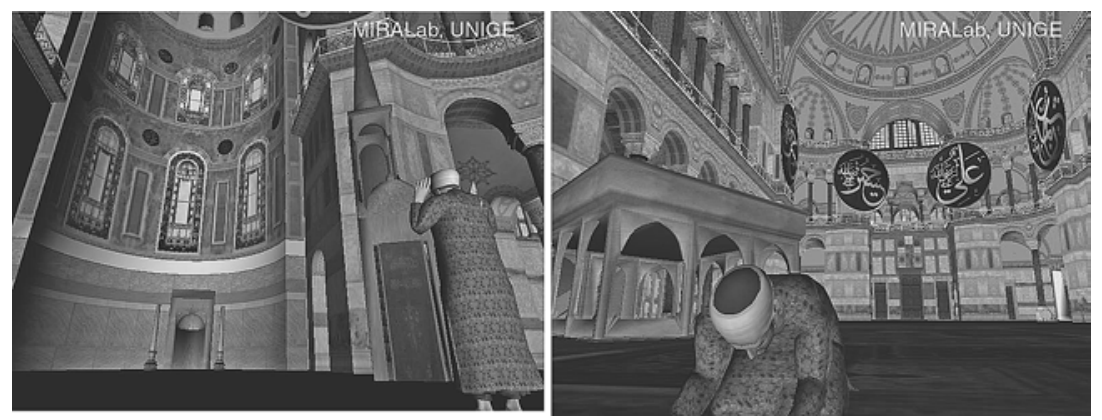

The Following screenshots illustrate a few examples of the Hagia Sophia fully real-time VR reconstructed edifice (as a 16th century mosque), which constitutes the only known, published effort of this kind and scale, aimed at the visualization of this particular cultural heritage site.

\section{Conclusions}

We are living in a world in which the arts, sciences and technology are becoming inextricably integrated strands in a new emerging cultural fabric [16]. Our knowledge of ourselves expands with the advent of new technologies that provide new tools for both communication and expression as well as a social context for daily experiences. Culture is in its broader sense a 'product' of our everyday life and experiences [17]. The actual records of culture are constituted from performances to artifacts that have been created in a persistent manner but inevitable decay. That is depicted in the notion of cultural heritage where it consists of what is called "Tangible Heritage" such as buildings, artifacts and media as well as the "Intangible Heritage" containing art expressions (music, dance, literature etc.), languages, folklore etc. Michael Chrichton in his novel Timeline [18] gives an example of a social context of the future where time travel to the past can be a unique mean of actually feeling experiences characterized by both pathos' ${ }^{\prime}$ and ethos ${ }^{2}$, integral connotations of the term cultural heritage.

Succeeding the virtual restitution of any complex heritage sites requires accurate choices for each phase of the modeling, texturing or lighting

'Pathos: I. that quality in speech, writing, music, or artistic representation (or in events, circumstances, persons, etc.), which excites a feeling of pity or sadness... 2. In reference to art, esp. ancient Greek art: The quality of the transient or emotional, as opposed to the permanent or ideal. Source: Oxford English Dictionary

${ }^{2}$ Ethos: I. the characteristic spirit, prevalent tone of sentiment, of a people or community; the 'genius' of an institution or system. 2. In reference to ancient ësthetic criticism and rhetoric. Aristotle's statement that Polygnotus excelled all other painters in the representation of 'ethos' app. meant simply that his pictures expressed 'character'; but as Aristotle elsewhere says that this painter portrayed men as nobler than they really are, some modern writers have taken ethos to mean 'ideal excellence.'... Source: Oxford English Dictionary

Time-Dependent Illumination and Animation of Virtual Hagia-Sophi 
processes and special attention must be used when the models have to be prepared for real-time platforms. Furthermore precise and reliable source data is critical for a scientifically correct and accurate restitution. Interpretative and comparative issues are also necessary when the restitution is targeting lost architectural elements of the heritage site. In the present article each step of the virtual inhabited restitution of Hagia Sophia in Istanbul, both as Byzantine cathedral and Islamic mosque, has been described: common problems, pitfalls, solutions and specific choices were illustrated in order to establish a complete methodology of bringing large, complex and endangered edifices to life and simulating historical characters as well as their architectural evolution.

Finally, the use of new information technologies, in conjunction and with the essential support of traditional sources and materials, has been presented in order to describe our approach to the restoration and renovation process targeted at ancient monuments. Hence with the utilization of the mentioned approach for the situations where architectural restoration and protection are not available, virtual restoration and conservation is exhibited. Such virtual heritage simulations are a fundamental aspect for the full understanding of the historical and social development of vast communities and form a 'virtual material witness' of the process of civilization.

\section{Acknowledgements}

The presented work has been supported by the Swiss Federal Office for Education and Science in frame of the EU INCO-MED CAHRISMA project.

\section{References}

I. Nandi, A., Marichal, X. Transfiction, proceedings of Virtual Reality International Conference, Laval, 2000.

2. Heidegger, M., Sein und Zeit, Max Niemeyer Verlag, Tübingen, 1993, p. 38 (translated according to the English translation of J. Macquarrie and E. Robinson Blackwell, 1962)

3. http://www.virtualheritage.net [14-2-2007]

4. Kim, Y., Kesavadas, T., Paley, S.M., Sanders, D.H., Real-time Animation of King Ashur-nasir-pal II (883-859 BC) in the Virtual Recreated Northwest Palace, in: VSMM200 I Proceedings, IEE Computer Society, Los Alamitos, 200I, pp. I28-I36.

5. De Leon, V., Berry, R., Bringing VR to the Desktop:Are you Game?, in: IEEE Multimedia, April-June, 2000, pp. 68-72.

6. Levy, R.M., Temple Site at Phimai: Modelling for the Scholar and the Tourist, in: VSMM200I Proceedings, IEE Computer Society, Los Alamitos, 200I, Pp. I247-I58.

7. Papagiannakis, G., L'Hoste, G., Foni, A., Magnenat-Thalmann, N., Real-Time Photo Realistic Simulation of Complex Heritage Edifices, in: VSMM200I Proceedings, 200I, Pp. 218-227.

8. Kato, S., Aoki, T., Hidaka, K., Nakamura, H., Finite-Element Modeling of the First and Second Domes of Hagia Sophia, in: Hagia Sophia, from the age of Justinian to the present, Cambridge University Press, 1992, pp 103-119.

9. Mark, R., Cakmak, A. S., Erdik, M., Preliminary Report on an Integrated Study of the Structure of Hagia Sophia: Past, Present, and Future, in: Hagia Sophia, from the age of Justinian to the present, Cambridge University Press, 1992, Pp |20-131.

Alessandro E. Foni, George Papagiannakis, Nedjma Cadi-Yazli and Nadia Magnenat-Thalmann 
10. Knöpfle, C., Virtual Cathedral of Siena, 2000, http://www.igd.fhg.de/igd-a4/ projects23.html [14-2-2007].

II. Nagakura,T., The DIGITARMA, presented at SIGGRAPH 98, Art and Multimedia SKETCH.

12. Kinross, HAGIA SOPHIA, The reader's Digest Association Limited, London, 1973.

13. Hoffmann,V., Die Hagia Sophia: Bilder aus sechs Jahrhunderten und Gaspare Fossatis Restaurierung der Jahre 1847 bis 1849, Peter Lang, Bern, 1999.

14. Mainstone, R. J., HAGIA SOPHIA Architecture, Structure and Liturgy of Justinian's Great Church, Ed. Thames and Hudson, 1988.

15. Ponder, M., Papagiannakis, G., Molet, T., Magnenat-Thalmann, N., Thalmann, D., VHD++ Development Framework: Towards Extendible, Component Based VR/AR Simulation Engine Featuring Advanced Virtual Character Technologies, in proceedings of Computer Graphics International, CGI, IEEE Computer Society Press, 2003, pp. 96-104.

16. Coyne, R., Technoromanticism, digital narrative, holism and the romance of the real, The MIT Press, Cambridge, Mass. 1999, pp. viii.

17. Salzburg Research Forschungsgesellschaft, Technological Landscapes for Tomorrow's Cultural Economy: Definitions-trends-hypotheses, http://www.salzburgresearch.at [14-2-2007].

18. Chrichton, M., Timeline, Alfred A. Knoph, New York, 1999.

Alessandro E. Foni, George Papagiannakis, Nedjma Cadi-Yazli and

Nadia Magnenat-Thalmann

University of Geneva

CUI, MIRALab

Site de Battelle

7, rte de Drize

I227 Carouge, Geneva

Switzerland

foni@miralab.unige.ch, Papagiannakis@miralab.unige.ch,

cadi@miralab.unige.ch, thalmann@miralab.unige.ch

Time-Dependent Illumination and Animation of Virtual Hagia-Sophia 
Chapter $06 \quad 16 / 7 / 07 \quad 12: 33$ pm Page 302

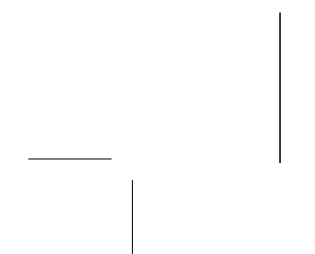




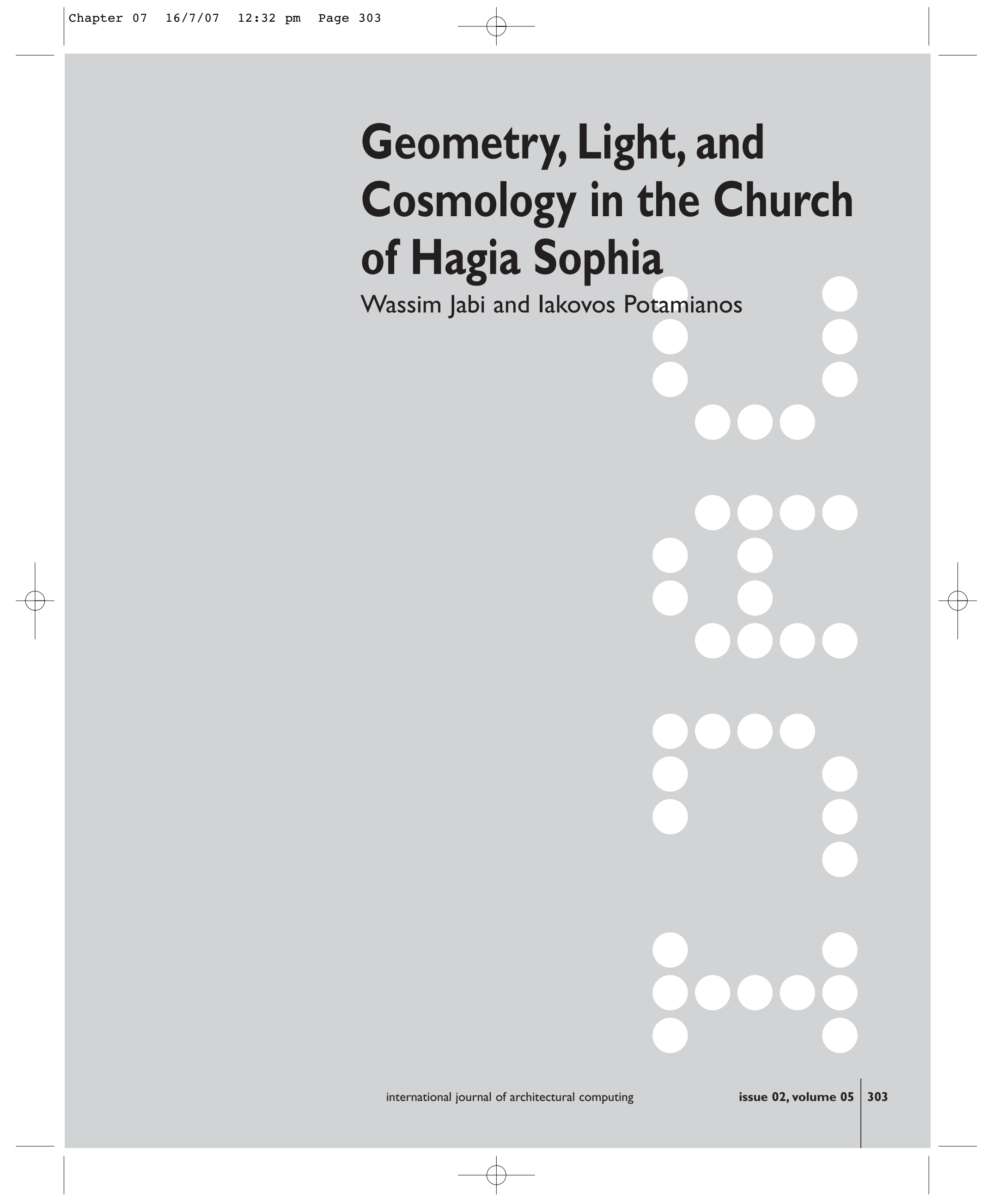

